\title{
Sistema de Desafios Abertos Inteligentes: um incremento computacional lúdico para se trabalhar o problema da aprendizagem
}

\author{
Othon C. Bastos Filho ${ }^{1}$, Margarete Axt ${ }^{1}$, Sofiane Labidi ${ }^{2}$, Paloma Dias Silveira ${ }^{3}$, Nilson dos \\ Santos Costa $^{2}$ e Luis Carlos C. Fonseca ${ }^{1}$ \\ ${ }^{1}$ Universidade Federal do Rio Grande do Sul (UFRGS) \\ Programa de Pós-Graduação em Informática na Educação - PPGIE \\ Av. Paulo Gama, 110 - prédio $12105-3^{\circ}$ andar sala 332 \\ 90040-060 - Porto Alegre (RS) - Brasil \\ Fone:(0xx51) 3316-3986 - Fax: (0xx51) 3316-3997 \\ othonb@pgie.ufrgs.br, othonb@ hotmail.com
}

2 Universidade Federal do Maranhão, Laboratório de Sistemas Inteligentes, LSI/UFMA, Campus do Bacanga, 65.000-000 - São Luís-MA, Brasil.

3 Laboratório de Estudos em Linguagem, Interação e Cognição, LELIC/UFRGS

Av. Paulo Gama, nº 100, FACED,8 andar, Centro - Porto Alegre (RS) - Brasil

Resumo - O trabalho contextualiza em linhas gerais ações da Tecnologia Educacional aplicando técnicas da Inteligência Artificial com a teoria de Jean Piaget. Tal pesquisa, ainda em fase de implementação, tem como hipótese que a partir de um incremento lúdico, apoiado pela IA, será possível alcançar situações de aprendizagem e, conseqüentemente, de desenvolvimento cognitivo para seu usuário. Isso se dá por meio de estágios e a adaptabilidade do sistema com o usuário, ou seja, o usuário interage com o sistema, tornado uma proposta de sistema construtivista, O IOCS faz parte de uma proposta de plataforma-ambiente integrada entre o Laboratório Sistemas Inteligentes LSI/UFMA e o Laboratório de Estudos em Linguagem, Interação e Cognição LELIC/UFRGS e permitirá uma visualização do relacionamento entre os processos mentais cognitivos e a emergência de emoções possibilitando ainda um suporte à docência e à formação continuada em ambientes informatizados.

Palavras-Chave: Estágios cognitivos, Abstração Reflexionante, Método Clínico, Multiagentes e Desafios Abertos.

Abstract - The work contextually in general lines action of the Educational Technology applying techniques of Artificial Intelligence with the theory of Jean Piaget. Such research, still in implementation phase, has as hypothesis that from a playful increment, supported for IA, will be possible to reach learning situations and, consequently, of cognitive development for its user. This if gives by means of periods of training and the adaptability of the system with the user, or either, the user interacts with the system, proposal of constructivist system. The IOCS is part of an platform-environment proposal integrated between the Laboratory Intelligent Systems - LSI/UFMA and the Laboratory of Studies in Language, Interaction and Cognition - LELIC/UFRGS and will still allow to a visualization of the relationship between the cognitive mental processes and the emergency of emotions making possible a support to the professor and the formation continued in computerization environments.

Keywords: Cognitivos periods of training, Reflexionante Abstraction, Clinical Method, Multiagentes and Open Challenges. 


\section{Introdução}

Hoje, várias instituições de ensino estão buscando a inclusão de seus alunos à educação digital, e o incremento lúdico, como os jogos, tem sido uma das estratégias utilizadas para se trabalhar os problemas relacionados à aprendizagem. Os "jogos" chamam a atenção pelo desafio dos estágios e pelas interfaces atraentes, e principalmente pela característica de jogo coletivo viabilizada pela conexão em rede e as possibilidades de cooperação grupal. Com isso, torna-se um importante recurso para atendimento a crianças com déficit de atenção, entre outras dificuldades. Entretanto, os jogos de uma maneira geral não são adaptativos, ou seja, não tem interação ou novas adaptações de ações externas e internas, já que as mesmas foram previamente codificadas e compiladas.

Pensando nessa última característica que veio a necessidade de se propor, não propriamente jogos, mas um sistema de desafios abertos inteligentes (IOCS - Intelligent Open Challenges System) (Bastos Filho, et al., 2004). Esta proposta está voltada para a educação, isto é, vem ao encontro de ofertar atividades que instiguem as construções de significados estruturados por meio da interação e do componente lúdico, e tragam, ao mesmo tempo, para os participantes, possibilidades de ganho cognitivo.

A idéia é trabalhar a aprendizagem a partir de uma concepção de construção do conhecimento, ou seja, a aprendizagem que ocorre por meio de situações desafiadoras. E, para atender a este pressuposto, utilizamos desafios 1 com regras lógicas incluídas na programação e com o aporte da tecnologia multiagentes vinda da Inteligência Artificial. Espera-se desafiar os usuários à problematização, motivando-os à interação necessária com os demais participantes de modo a chegar a soluções interessantes a partir do proposto pelo sistema. Como desfeche dos desafios os participantes, além da discussão conjunta das possíveis alternativas de solução, possam explicitar as soluções encontradas, deixando-as registradas, bem como possam interagir com vistas a propor coletivamente novas possibilidades em termos de desafios.

Os desafios estão baseados em estágios, a partir das provas piagetianas, ou seja, buscam e incentivam a compreensão dos mecanismos envolvidos na construção de conhecimento. No Sistema Inteligente pretende-se uma adaptação do método clínico para a concepção e operacionalização do sistema, aplicando-se os desafios e, com isto, observando-se o processamento da construção realizada pelo usuário, assim como o entendimento de suas próprias soluções.

O IOCS deverá compor um "pool" de softwares de uma nova plataformaambiente que é a integração do aplicativo NETCLASS ${ }^{2}$ desenvolvido pelo Laboratório Sistemas Inteligentes - LSI/UFMA (Labidi et al., 2000) e com o aplicativo CIVITAS ${ }^{3}$ do Laboratório de Estudos em Linguagem, Interação e Cognição - LELIC/UFRGS, (Axt et al., 2000).

\section{Sistema de Desafios Abertos Inteligentes -}

Este artigo apresenta uma proposta que está em fase de implementação, e propõe um sistema diferente na aplicação dos multiagentes, porque enfatiza a pró-atividade nos dois sentidos, isto é, a interação, o sujeito (usuário) com o objeto (sistema), criando situações de estratégias desafiadoras abertas baseadas em provas e adaptações da metodologia piagetiana, trabalhando assim, o entendimento e a construção de patamares de conhecimento.

\footnotetext{
${ }^{1}$ Para esta pesquisa os desafios possuem predicativos de objetos de aprendizagem.

${ }^{2}$ NETCLASS é um ambiente ensino-aprendizagem que utiliza técnicas de inteligência artificial.

${ }^{3}$ CIVITAS é um software e metodologia de criação de uma cidade virtual em cooperação. 
Para Piaget, o desenvolvimento do conhecimento é concebido por quatro fatores: o primeiro a maturação, o segundo o papel da experiência, o terceiro a transmissão social e o quarto ele chamou de equilibração. Todos são de suma importância, mas o foco deste artigo encontra-se no fator experiência, dos efeitos do ambiente físico na estrutura da inteligência e no fator equilibração, processos que se regulam a si próprios mediante uma compensação por compensação progressiva do sistema, isto é, um processo ativo.

A partir deste pressuposto teórico, o Sistema de Desafios Abertos Inteligentes IOCS (Intelligent Open Challengen System) é formado por duas situações:

O Sistema - Arquitetura desenvolvida usando a linguagem JavaTM (Java, 2004) e que tem como sua função principal a aplicação dos desafios, simulando assim, o papel do experimentador ${ }^{4}$, que será comentado mais detalhadamente no item a seguir.

Os Desafios - são programados na linguagem Action Script (Action Script, 2004), com predicativos de objetos de aprendizagem ${ }^{5}$, os quais irão formar um banco de desafios a ser usado pelo sistema, sendo eles baseados nos exames de Piaget encontrados na teoria Abstração Reflexionante (Piaget, 1995).

O Sistema de Desafios Abertos Inteligentes, IOCS - (Intelligent Open Challenges), acaba por constituir um sistema multiagentes que apóia os processos de aprendizagem, e sua utilização, assim se espera, é viável não somente em ambientes virtuais ou em outros (STI) ${ }^{6}$, mas também como apoio para o desenvolvimento de estratégias pedagógicas e também psicopedagógicas.

\subsection{O Sistema de Desafios Abertos Inteligentes}

A concepção teórico-educacional desse Sistema tem como base a teoria de Jean Piaget, principalmente a Abstração Reflexionante, além disso, a metodologia de pesquisa utilizada: o Método Clínico (Piaget, 95).

Para isso, pretende-se partir de tal teoria para orientar a concepção e a operacionalização do sistema multiagentes, trabalhando com o sujeito epistêmico e psicológico, desenvolvendo assim uma nova perspectiva de sistema inteligente para a educação.

Os resultados esperados devem enfatizar a pró-atividade nos dois sentidos, isto é, tanto do sistema como do seu usuário, criando situações de estágios estratégicos, por meio de desafios abertos, aferindo ao usuário um progresso significativo na sua capacidade cognitiva, propiciando a compreensão e a construção de novos patamares de conhecimento.

Para isso é necessário desenvolver abstrações cada vez mais reflexionantes, as quais se apóiam "sobre objetos procedente de ações ou operações do sujeito e transferindo a um plano superior [de organização] tudo o que foi tirado de um nível inferior de atividade" (Piaget, 1995), produzindo novas formas de conhecimento.

A abstração reflexionante comporta sempre em dois aspectos:

A reflexão - como ato mental de reconstrução e reorganização sobre o patamar superior daquilo que foi assim transferido do inferior.

O reflexionamento - transpõe-se a um plano superior o que colhe no patamar precedente;

\footnotetext{
${ }^{4}$ Experimentador é quem aplica o método clínico e desenvolve as seguintes ações: saber observar, ou seja, deixar a criança falar, não desviar nada, não esgotar nada e saber buscar algo de preciso, como uma tarefa com hipóteses pré-determinadas, propondo contra-argumentações, perguntas de antecipações etc

5 Objeto de Aprendizagem é qualquer recurso digital que possa ser reutilizado e ajude na aprendizagem.

${ }^{6}$ STI - Sistema de Tutores Inteligentes, ou (Intelligent System Tutor - ITS)

V. $3 \mathrm{~N}^{\circ} 1$, Maio, 2005
} 
Na figura 01 o processo de abstração reflexionante, formando os patamares.

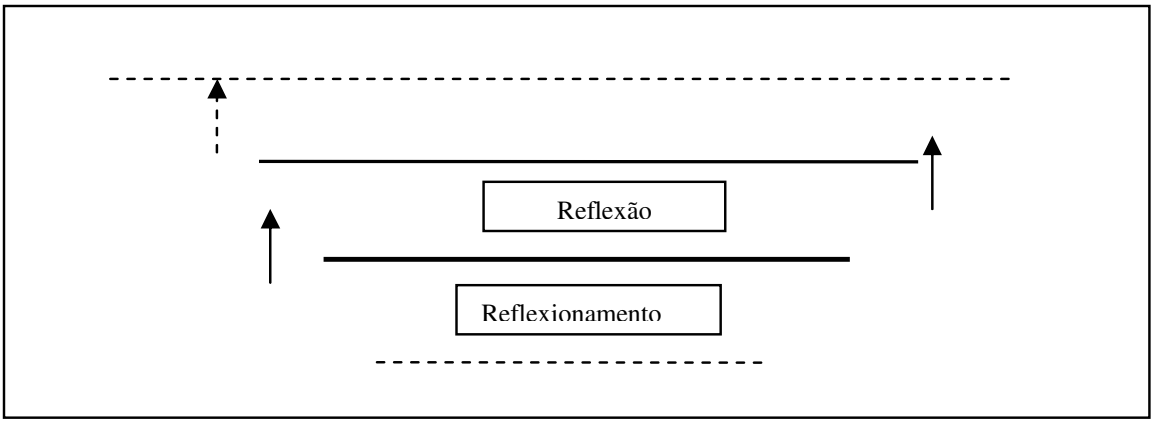

Figura 01 - Processo da Abstração Reflexionante

Tais formas abstraídas ou depreendidas a partir das atividades cognitivas do sujeito (esquemas ou coordenações de ações, operações, estruturas, etc.), pela retirada de certos caracteres mais constantes, são, por sua vez, utilizadas para outras finalidades (novas adaptações, novos problemas).

Há outro fator importante do sistema que está em consonância com os padrões do Método Clínico Piagetiano: a possibilidade de uma aplicação a objetos de aprendizagem junto a uma metodologia de estudo cognitivo, simulando-se o papel do experimentador no método clínico, com o objetivo de construção de novos patamares, inclusive, por tomadas de consciência.

"O bom experimentador deve, efetivamente, reunir duas qualidades muitas vezes incompatíveis: saber observar, ou seja, deixar a criança falar, não desviar nada, não esgotar nada e, ao mesmo tempo, saber buscar algo de preciso, ter a cada instante uma hipótese de trabalho, uma teoria, verdadeira ou falsa, para controlar." (Piaget, 1993, p. 17)

E por último o sistema possui três estágios: estágio de observação, estágio de fazer para compreender e estágio de compreender no pensamento. Com uma seqüência estruturada em níveis. Isto é, não é possível alcançar o segundo nível a não ser que o equilíbrio tenha sido alcançado no primeiro nível, e o equilíbrio do terceiro nível só se torna possível quando o equilíbrio do segundo tenha sido alcançado, e assim por diante.

\subsection{Desafios Abertos}

Os desafios são objetos de aprendizagem e foram divididas em três fases:

- Abstração das relações lógico-aritméticas - neste desafio é trabalhado o raciocínio sobre hipótese independente do conteúdo, que têm, como elementos básicos, quatro transformações chamadas INRC - (Iidentidade, N-negação, R-reciprocidade e C-correlativa).

- Abstrações da ordem das relações espaciais - Fazem parte das estruturas lógico-aritméticas e são formas elementares de seriações ou de sucessões de movimentos, de maneira a precisar as perspectivas enquanto parte das abstrações. Ou seja, há uma seqüência, e para que o usuário possa solucionar os desafios, terá que entendê-la, bem como a lógica aplicada, enquanto parte do processo de abstração reflexionante. Possibilitando uma observação da construção cognitiva do usuário, por meio de suas ações. 
- Abstração das relações espaciais - Neste ítem será reproduzido a existência de um espaço dos objetos, como também uma geometria do sujeito utilizando diagonais, certos movimentos cíclicos e diversas formas de translações e de rotações. Para se reproduzir estas formas o mais próximo da realidade, será proposto o uso de linguagem de programação que tenha capacidade de gerar movimentos em 3D, como: VRML (Virtual Reality Model Language)(VRML, 2005) ou Java 3D(Java, 2005).

\section{Arquitetura do IOCS}

Nesta seção, apresentaremos a arquitetura do IOCS, esta se inspira no método clínico piagetiano, onde existe o papel do experimentador, especialista que aplica os testes. Na proposta do IOCS, este experimentador será simulado pelos agentes inteligentes que buscam padrões no seu usuário para criar estratégias desafiadoras. Essa plataforma será desenvolvida usando a linguagem Java (JAVA, 2004).

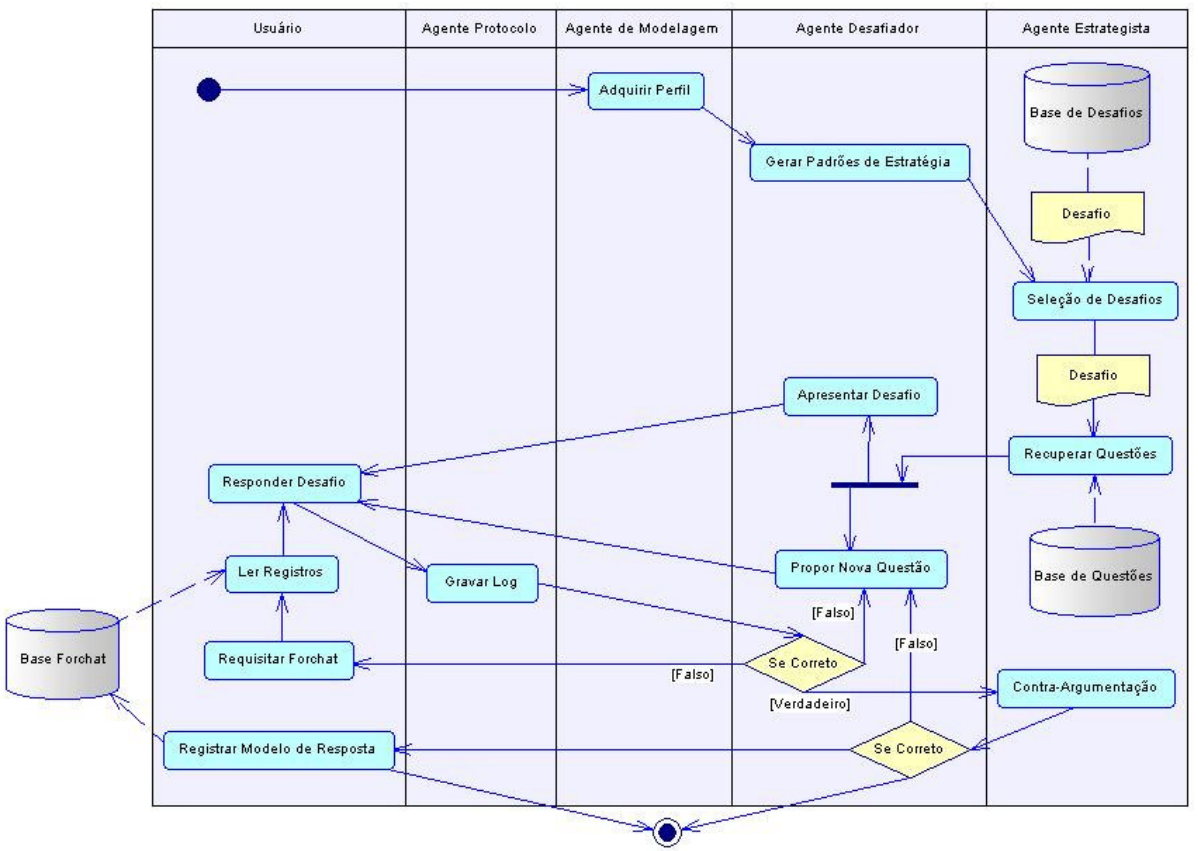

Figura 02- Arquitetura IOCS

Agente de Modelagem - É a ligação entre o usuário e o sistema. Baseado na informação da interação do usuário com o sistema, ou seja, trabalha com os 5 tipos de reação observados pelo exame clínico piagetiano. Esta informação é fator de decisão para o agente estrategista na escolha dos desafios.

Agente Estrategista - Reúne e situa funcionalidades de controle, ação estratégica. Informa ao agente de desafios a necessidade de um certo desafio baseado na informação processada do agente modelador e requisita à base de questões perguntas que levem a provocar desequilíbrios cognitivos no usuário, criando um processo de problematização para o desafio usado naquele momento, com isto, o sistema tenta simular o papel do experimentador. É também responsabilidade deste agente uma contra argumentação sobre a resposta que o usuário, no final do processo, entende como correta. 
Agente Desafiador - Responsável em apresentar os desafios e as questões elicitadas pelo agente estrategista ao usuário do sistema e apresentar o desafio ao usuário.

Agente de Protocolo - É a entidade responsável por registrar as ações do usuário no sistema durante o processo de solução do desafio, possibilitando um mapeamento da evolução cognitiva do usuário.

Base de Desafios - Local de armazenamento dos desafios e seus estágios, os quais serão acionados em consonância com as requisições do agente estrategista.

Software Forchat - Software que ficará disponível para utilização ou não pelo usuário do IOCS. Tem o intuito de produzir contribuições que levem à abstrações reflexionantes, a partir das contribuições dos usuários anteriores e seus desafios. Para isso, reúne, ao mesmo tempo, as propriedades de um chat - interatividade e ícones para denominar os participantes - e de um fórum - permanência das discussões possibilitando interações síncronas e assíncronas (AXT et al. 2000).

Usuário - É o agente humano do sistema, ele interage com o agente de modelagem, registra no software Forchat a sua experiência e compreensão obtidas das suas próprias soluções, caso quiser, podendo ser usada como auxílio por outros usuários on-line ou off-line caso os mesmos achem interessante. Dessa forma, o usuário faz parte integrante do sistema IOCS, além de ser o seu próprio usuário.

Base de Questões - Base de armazenamento de perguntas provenientes das provas piagetianas como: "O que coloquei lá? - Como são ordenadas? - Eu tenho mais flores ou mais rosas? - O que você observa?”. O Agente Estrategista irá requisitá-las conforme os padrões e pesos dados pela programação contidos no sistema e serão aplicadas ao usuário. Cada grupo de questões tem um nível de associação com os estágios dos desafios, com isso, não haverá as repetições de questões.

\section{Especificação do Sistema}

Algumas visões em termos de modelagem foram criadas baseado em Piaget para o Sistema de Aprendizagem Proposto. Os casos de uso e diagramas foram criados por meio da linguagem UML (Linguagem de Modelagem Unificada). O sistema foi dividido em três casos de uso, são eles: "Criação da Modelagem do Usuário", "Escolha de Estratégia", "Realizar Desafios". Cada um desses, "Casos de Uso" complementa as ações tomadas pelo sistema para a realização das atividades que permitem avaliar e validar o usuário. Sendo que para alimentar as decisões tomadas por esses agentes, foram criadas duas bases de dados, a "Base de Desafios" e a "Base de Estratégia". A visão geral dos casos de uso é mostrada na figura 03 .

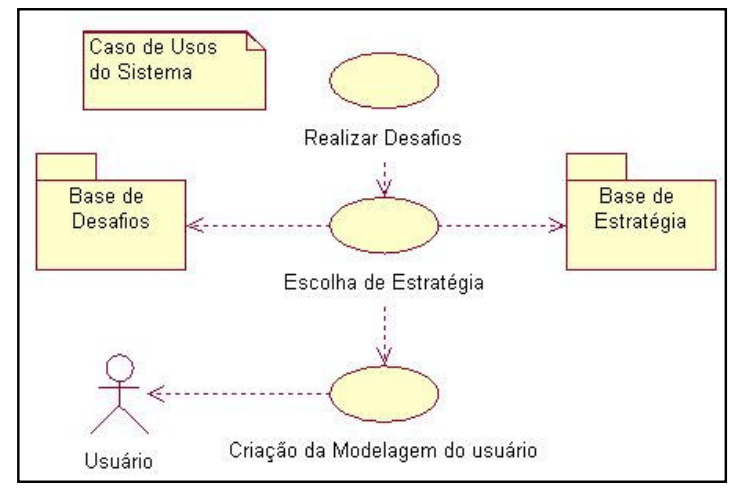

Figura 03 - Casos de uso do Sistema 
O caso de uso "Criação da Modelagem do Usuário", possui a responsabilidade de interação com o usuário para a obtenção de informações que sirvam de indicativo ou dados para a criação de um perfil do usuário. A criação da modelagem desse usuário poderá ser feita ou realizada utilizando técnicas de redes neurais ou simplesmente interagindo (sistema) com o usuário por meio de perguntas (neste caso de uso é considerado que o sistema interage com o usuário para criar o seu perfil). Estas informações do perfil desse usuário são posteriormente enviadas ao agente estrategista.

As informações baseadas na teoria de Piaget para a criação do perfil do usuário trabalham com os 5 tipos de reação observados pelo exame clínico (Piaget, 1926):

- Não importismo - quando não provoca esforço algum de resposta;

- Fabulação - expressada quando o usuário responde sem refletir, inventando uma situação.

- Crença Sugerida - quando a pergunta é sugestiva ou quando o usuário responde sem refletir influenciado pela sugestão do sistema;

- Crença Desencadeada - necessariamente influenciada pelas questões que o sistema irá fazer, pois a partir delas o usuário se vê forçado a raciocinar em certa direção e a sistematizar o seu conhecimento, ou seja, produto original do pensamento do usuário.

- Crença Espontânea - aparece quando a pergunta não é nova para o usuário, sendo a resposta fruto de uma reflexão anterior e original (abstração reflexionante), ou seja, desenvolvida pelo próprio usuário.

Baseado nesses itens a figura 04 mostra o agente de modelagem realizando a absorção das informações do usuário por meio dos itens baseados e advindos de Piaget.

De acordo com a análise do diagrama de classe da figura 03, o agente de modelagem (entidade de controle) controla as classes envolvidas em obter o perfil do usuário, em uma relação de dependência. Sendo que dependendo do usuário, essas informações podem vir de todas as classes de armazenamento (Não importimos, Fabulação, Crença Sugerida, Crença Desencadeada, Crença Espontânea) ou de algumas delas, ou pelo menos uma delas.

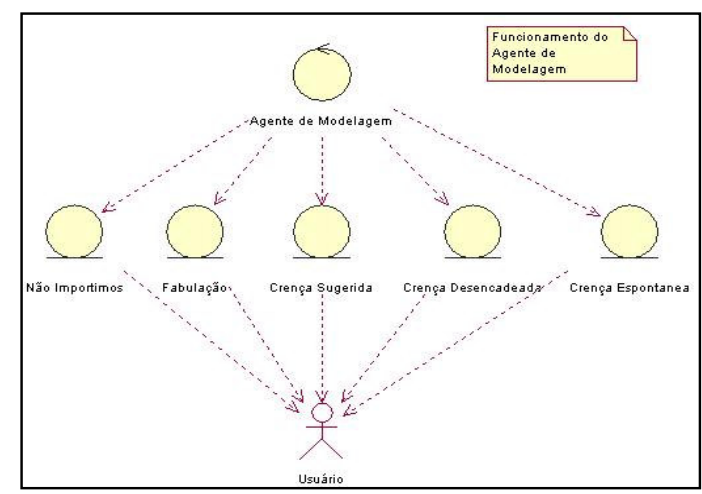

Figura 04 - Funcionamento Criação do Agente de Modelagem

Todas as Classes de armazenamento que irão absorver as informações do usuário dependem diretamente dele, as setas em direção ao usuário representam essa situação de dependência.

$\mathrm{Na}$ figura 05 observa-se o diagrama de colaboração da "Modelagem do Agente", o agente de controle de modelagem do agente, inicialmente carrega as informações do Banco de desafios, escolhido o desafio o controle do agente envia ao usuário por meio de uma interface do sistema para o usuário o desafio. 
Esse usuário preenche as respostas a esse desafio, após essa etapa, o agente por meio do seu centro de controle analisa as informações coletadas advindas do usuário para em seguida armazenar essas informações em uma base de informações do usuário que servirá de histórico do usuário. Lembrando que essas informações serão arquivadas por meio de um outro agente do sistema denominado de "Agente de Protocolo". Após a realização do arquivamento dessa informação por meio do agente de Protocolo, a mesma informação advinda do usuário será transmitida ao agente estrategista.

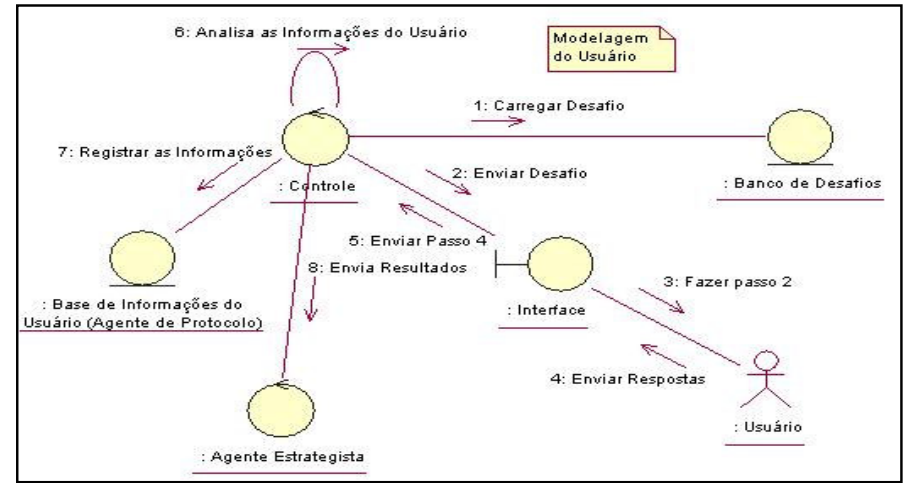

Figura 05 - Diagrama de Colaboração do caso de uso "Modelagem do Usuário"

O Agente estrategista (figura 06) de posse dessas informações provenientes do Agente de modelagem, cuja responsabilidade é a de colher as informações do usuário, realiza as operações básicas de verificação de usuário bem como recuperação das informações do usuário, cujo local de deposito dessas informações é a "Base de Informações do Usuário". De posse dessas informações o controle do agente de estratégia escolhe a melhor estratégia, essa estratégia está armazenada no banco de estratégia. Carregada a estratégia o passo seguinte será carregar o desafio, cuja gerencia e responsabilidade é o agente de desafio.

Realizado as operações de escolha de estratégia e de desafio, o controle do agente envia as informações para serem aplicadas ao usuário.

É importante frisar que todos os diagramas (classe, colaboração e outros) utilizados na elaboração do sistema possuem cenários alternativos no qual o sistema possui outros modos de alcançar os seus objetivos ou mesmo reiniciar os passos anteriores com outros cenários no qual possam alcançar os seus objetivos iniciais. Uma analogia a esse processo seria a utilização do procedimento de decisão "SE" da programação de computadores. Isto é, caso uma etapa não funcione a contento, outras ações tentarão conseguir retornar ao objetivo inicial de alcançar o objetivo. 


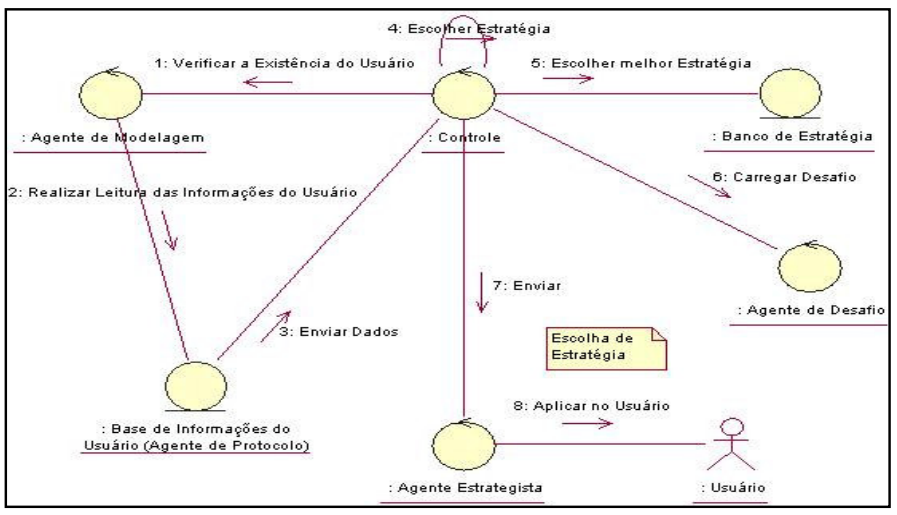

Figura 06 - Diagrama de Colaboração do caso de uso "Escolha de Estratégia"

Em outras palavras o objetivo do caso de uso por meio do agente estrategista é aplica a melhor ação estratégica para que o usuário possa ser testado, sendo que nesse momento o sistema como um todo está assumindo o papel de experimentador.

O ultimo caso de uso foi nomeado de "Realizar Desafios", cujo objetivo é fazer com que o agente desafiador possa selecionar ou busca o desafio na base de desafios, observando que tais desafios estão armazenados na base de desafios (que são constantemente atualizados) sendo tais desafios são requisitados pelo agente de Desafio.

De acordo com o diagrama de colaboração da figura 07, o agente de desafios recebe as informações do agente de estratégia para depois realizar uma análise dessas informações a serem realizadas pelo controle do agente. Analisadas as informações o desafio é carregado do "Banco de Desafios". Carregado o desafio o passo seguinte é enviar para a interface do sistema o desafio para o usuário. Ao usuário respondendo ao desafio, vários procedimentos são disparados para a análise da resposta do usuário, não tendo como único parâmetro o acerto ou erro ao desafio. Pois vários valores são avaliados de acordo com as teorias de Piaget como, por exemplo, a Fabulação.

A interface recebendo as respostas do usuário, as transmite ao controle do agente de desafios. A resposta é analisada pelo centro de controle do agente, realizada esta ação, o passo 9 envia uma ordem para grava as informações obtidas do usuário.

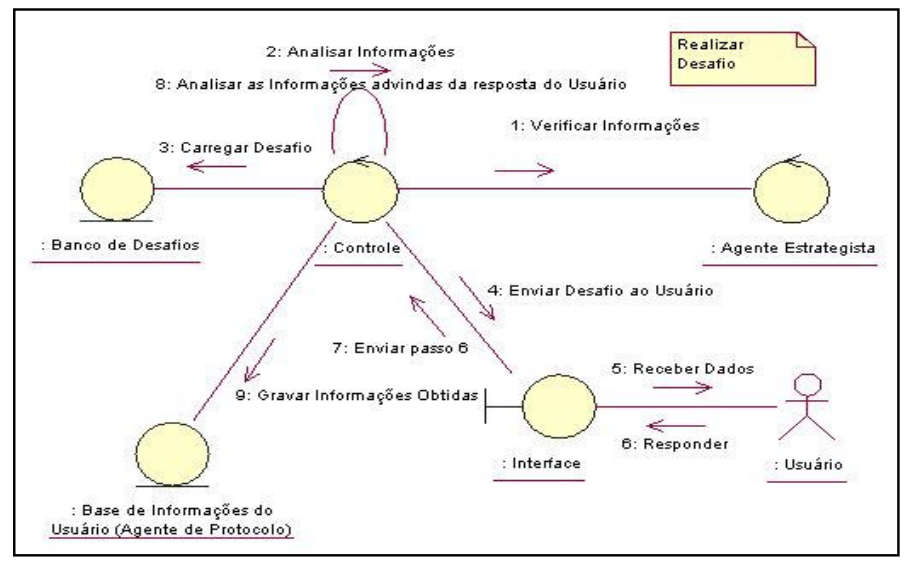

Figura 07 - Diagrama de Colaboração do caso de uso "Realizar Desafio"

Essas informações alimentam a "Base de Informações do usuário", cuja responsabilidade de gerenciar de armazenar essas informações é do agente de protocolo. 
Essas bases de informações não são estáticas, elas são constantemente atualizadas de acordo com as respostas do usuário a cada novo desafio e reação a cada um deles. Cada estratégia e desafio são escolhidos de acordo com as características individuais de cada usuário do sistema.

Diferente de outros sistemas que cuja preocupação é a de verificar somente se o usuário acertou ou não a questão solicitada. O sistema de aprendizagem proposto coloca em pratica teorias básicas ensinadas e divulgado pela pedagogia, no qual existe uma real preocupação do porque e do como se realiza o processo de aprendizagem.

\section{Conclusão}

A proposta desta pesquisa contextualizada neste artigo é buscar por meio de um incremento lúdico um desenvolver de atividades ligadas a aprendizagem, promovendo o desenvolvimento do conhecimento como um processo espontâneo, ou seja, um processo com auto-regulação. Para isso, o sujeito tem que ser ativo interagindo com o objeto, conseqüentemente defrontar-se-á com uma perturbação externa e interna, e reagirá como fim de compensar e tenderá para o equilíbrio como processo ativo. Este processo de equilibração toma forma de uma sucessão de níveis de equilíbrios, situação fundamental trabalhada pelo sistema e pelos objetos de aprendizagem (desafios), e Piaget chama de fator de equilibração (Rippler, R e Rockcastle, V, p. 7-19, 1964).

Outra situação é que o sistema irá mapear a ação do usuário registrando a evolução do ganho cognitivo do mesmo. Também, disponibilizará situações para ele mostra como chegou ao seu entendimento, possibilitando uma resposta pessoal e aberta, já que ele irá expressar o que entendeu, não se preocupando se esta certo ou errado.

Finalmente, o que se pretende é desenvolver um sistema inteligente, adaptativo e construtivista, propiciando ao sujeito o inicio da a ação sobre o objeto e não somente a idéia de estímulo-resposta, ou seja, que o estímulo se torne significativo a partir do momento que há uma estrutura permitindo sua assimilação.

\section{Bibliografia}

ACTINSCRIPTS, 2004 - http://www.actionscripts.org/

AXT, M. Tecnologia na Educação, Tecnologia para Educação: um texto em construção. Informática na educação: teoria \& prática, v. 3, n. 1, p. 51-62, set. 2000).

BASTOS FILHO, Othon. C., AXT, Margarete, FONSECA, Luís Carlos, LABIDI, Sofiane, GUIMARÃES, Lucas Nunes, THOMAZ, Andrei Rubina, NASCIMENTO, Edson. Jogos inteligentes como sistemas abertos: singularizando as possibilidades de interação com desafios lógicos. Revista Novas Tecnologias na Educação, ISSN 1679-1916,2004. http://www.cinted.ufrgs.br/renote/nov2004/artigos/othon_jogos_inteligentes.pdf.

JAVA, 2005. http://java.sun.com/

LABIDI, S; SILVA, J. C.; COUTINHO, L.R.; COSTA, E. B. Agent-based tutoring system for supporting cooperative and distant learning. In Proceedings of the International Conference on Computers and Advanced Technology in Education (CATE'2000).

MONTANGERO, J., and MAURICE-NAVILLE, D.. Piaget ou l'intelligence en marche. Liège, Mardaga, (1994).

PIAGET, Jean, A representação do mundo na criança. Rio de Janeiro: Record, 1926. 
PIAGET, Jean. A Equilibração das Estruturas Cognitivas. Problema central do desenvolvimento. Trad. Álvaro Cabral. Rio de Janeiro: Zahar, 1976a.

PIAGET, Jean. A Formação do Símbolo na Criança. Imitação, jogo e sonho, imagem e representação.Trad. Alvaro Cabral. Rio de Janeiro: Zahar, 1971.

PIAGET, Jean. Abstração Reflexionante: Relações lógico-aritméticas e ordem das relações espaciais. Trad. Fernando Becker e Petronilha G. da Silva, Porto Alegre: Artes Médicas, 1995.

PIAGET, Jean. Da Lógica da Criança à Lógica do Adolescente. São Paulo: Pioneira, 1976b.

PIAGET, Jean. Fazer e Compreender. Trad. Cristina L. de P. Leite. São Paulo: Melhoramentos; EDUSP, 1978. 186p.

PIAGET, Jean; et alii. A Tomada da Consciência. Trad. Edson B. de Souza. São Paulo: Melhoramentos e EDUSP, 1977. 211p.

RIPPLE, R e ROCKCASTLE, V. Piaget rediscovered. Cornell University, 1964

VRML, 2004. Full details of the history and specifications can be seen at the VRML repository at http://www.sdsc.edu/vrml/. 